\title{
Surgical treatment of low lumbar osteoporotic vertebral collapse: a single-institution experience
}

\author{
Hideaki Nakajima, MD, PhD, Kenzo Uchida, MD, PhD, Kazuya Honjoh, MD, Takumi Sakamoto, MD, \\ Makoto Kitade, MD, and Hisatoshi Baba, MD, PhD
}

Department of Orthopaedics and Rehabilitation Medicine, Faculty of Medical Sciences, University of Fukui, Japan

\begin{abstract}
OBJECTIVE Low lumbar osteoporotic vertebral collapse (OVC) has not been well documented compared with OVC of the thoracolumbar spine. The differences between low lumbar and thoracolumbar lesions should be studied to provide better treatment. The aim of this study was to clarify the clinical and imaging features as well as outcomes of low lumbar OVC and to discuss the appropriate surgical treatment.
\end{abstract}

METHODS Thirty patients ( 10 men; 20 women; mean age $79.3 \pm 4.7$ years [range $70-88$ years]) with low lumbar OVC affecting levels below L-3 underwent surgical treatment. The clinical symptoms, morphological features of affected vertebra, sagittal spinopelvic alignment, neurological status before and after surgery, and surgical procedures were reviewed at a mean follow-up period of 2.4 years.

RESULTS The main clinical symptom was radicular leg pain. Most patients had old compression fractures at the thoracolumbar level. The affected vertebra was flat-type and concave or $\mathrm{H}$-shaped type, not wedge type as often found in thoracolumbar OVC. There were mismatches between pelvic incidence and lumbar lordosis on plain radiographs. On CT and MR images, foraminal stenosis was seen in 18 patients $(60 \%)$ and canal stenosis in 24 patients $(80 \%)$. Decompression with short fusion using a posterior approach was performed. Augmentations of vertebroplasty, posterolateral fusion, and posterior lumbar interbody fusion were performed based on the presence/absence of local kyphosis of lumbar spine, cleft formation, and/or intervertebral instability. Although the neurological and visual analog scale scores improved postoperatively, 8 patients $(26.7 \%)$ developed postoperative complications mainly related to instrumentation failure. In patients with postoperative complications, lumbar spine bone mineral density was significantly low, but the spinopelvic alignment showed no correlation when compared with those without complications.

CONCLUSIONS The main types of low lumbar OVC were flat-type and concave type, which resulted in neurological symptoms by retropulsed bony fragments generating foraminal stenosis and/or canal stenosis. For patients with low lumbar OVC, decompression of the foraminal and canal stenosis with short fusion surgery via posterior approach can improve neurological symptoms. Since these patients are elderly with poor bone quality and other complications, treatments for both OVC and osteoporosis should be provided to achieve good clinical outcome.

http://thejns.org/doi/abs/10.3171/2015.4.SPINE14847

KEY WORDS osteoporotic vertebral collapse; low lumbar spine; clinical feature; surgery; clinical outcome

$\mathrm{T}$ HE incidence of vertebral body fracture associated with osteoporosis increases as the population ages. ${ }^{2,12}$ Most cases can be managed conservatively, but cases with neurological complications should be treated surgically to allow patients early return to daily life activities. About $30 \%$ of patients with vertebral body fracture present with progressive collapse, $13 \%$ show vertebral nonunion, and 3\% develop osteoporotic vertebral collapse (OVC) with neurological deficits. ${ }^{25}$ Osteoporotic vertebral fractures commonly affect the thoracolumbar spine; however, they are also often found in low lumbar spine levels. In this regard, a large number of patients have been reported with thoracolumbar osteoporotic vertebral fractures and collapse, and various treatment modalities have been described to date, including both surgical and conservative therapies. ${ }^{5,10}$ However, there are only a few reports on treatment of patients with low lumbar OVC.

Lesions at the thoracolumbar junction manifest specific neurological symptoms since that level of the spinal column contains a complex of nerve structures, such as the

ABBREVIATIONS BMD = bone mineral density; JOA = Japanese Orthopaedic Association; $\mathrm{LL}=$ lumbar lordosis; $\mathrm{OVC}=$ osteoporotic vertebral collapse; $\mathrm{PI}=$ pelvic incidence; PLF = posterolateral fusion; PLIF = posterior lumbar interbody fusion; PSF = pedicle screw fixation; PT = pelvic tilt; PTH = parathyroid hormone; $S S=$ sacral slope; VAS = visual analog scale; $\mathrm{VCR}=$ vertebral column resection; $\mathrm{VP}=$ vertebroplasty.

SUBMITTED August 17, 2014. ACCEPTED April 10, 2015.

INCLUDE WHEN CITING Published online September 18, 2015; DOI: 10.3171/2015.4.SPINE14847. 
spinal cord, conus medullaris, and cauda equina. The objective neurological symptoms caused by thoracolumbar OVC do not only include motor weakness but also urinary disturbance and/or significant sensory disturbance. On the other hand, the low lumbar spine presents lordotic alignment, and the spinal column contains only cauda equina. Because of these anatomical features, no neurological complications are encountered in low lumbar OVC, and the rate of surgical indication may be lower compared with thoracolumbar lesions. However, it is also important to study other differences between low lumbar OVC and thoracolumbar lesions: clinical status, neurological symptoms, radiological findings, and surgical strategies.

Surgical treatment of the osteoporotic spine is challenging, and various surgical procedures using a variety of techniques have been advocated in the management of OVC. Importantly, most patients who receive surgical treatment are elderly, and prolonged surgical stress may involve risks. Therefore, the important question of how to improve surgery for OVC remains somewhat unanswered. Especially for low lumbar OVC, the same treatment used for thoracolumbar lesion might not be suitable. Posterior decompression surgery alone is not sufficient for these patients because neurological symptoms may result from retropulsed bony fragments and vertebral instability. Watanabe et al. ${ }^{29}$ evaluated the surgical results of lumbar spinal canal stenosis associated with compression fracture at L3-5 and reported that the mean recovery rate of the Japanese Orthopaedic Association (JOA) score at final follow-up was $50.4 \%$ in patients who underwent posterior decompression surgery and $66.7 \%$ in patients who underwent posterior intervertebral fusion surgery.

In the present study, we describe the clinical and radiological features and outcome of 30 consecutive patients with low lumbar OVC and associated neurological symptoms who underwent surgical treatment. Intervertebral cleft is a highly suggestive sign of osteonecrosis and has dynamic mobility indicating instability within the fracture. Because additional support of the anterior column might be necessary to provide stabilization and pain relief, we stratified these patients by the presence of cleft formation. The primary purpose of this study was to discuss the appropriate choice of surgical treatment and outcome for low lumbar OVC. For this purpose, we examined the differences in clinical and imaging features and associated neurological symptoms of low lumbar OVC compared with those of thoracolumbar lesions.

\section{Methods}

\section{Study Design, Inclusion, and Exclusion}

We retrospectively reviewed the medical records of 123 patients who underwent surgeries for OVC between 1993 and 2013 at our university medical center. Among these patients, we enrolled 30 patients (10 men and 20 women) (24.4\%) whose affected vertebral levels were below L-3. The affected vertebrae were L-3 collapse in 12 patients, L-4 in 13, and L-5 in 5. The mean age at surgery was 79.3 \pm 4.7 years (range $70-88$ years). All 30 patients initially received conservative treatment, but neurological symptoms subsequently developed and/or vertebral collapse progressed gradually. Of these 30 patients, cleft formation was identified on the radiographs and MR images at the affected vertebra in 14 patients. None of the patients had comorbidities that directly caused sensory or motor deficits in the lower extremities.

\section{Surgeries}

The surgeries for patients with cleft formation at affected vertebra $(n=14)$ were vertebroplasty $(V P)$ in 2 patients and the combination of decompression, VP, pedicle screw fixation (PSF), and posterolateral fusion (PLF) in 12 patients. We have favored since approximately 2005 a method by which vertebral reconstruction was performed with hydroxyapatite blocks or calcium phosphate cement filling via the pedicle of the fractured vertebra and supplemented by fixation of the vertebrae above and below with PSF. For patients without cleft formation $(n=16)$, we performed decompression, PSF, and PLF in 10 patients, and posterior lumbar interbody fusion (PLIF) in 4 patients. In 2 cases (cleft formation was identified in 1 patient), correction osteotomy (Ponte osteotomy, pedicle subtraction osteotomy, and vertebral column resection [VCR]) was performed. The 2 senior surgeons (H.B. and K.U.) performed all surgeries using a uniform technique. To avoid bias, the same surgeons were excluded from postoperative evaluation.

\section{Outcomes}

The clinical symptoms, low-back pain, radicular leg pain, and cauda equine sign, were assessed and stratified by the presence of cleft formation. The neurological status of each patient was assessed according to the JOA scoring system, and the postoperative neurological improvement rate was calculated using the following formula: (postoperative follow-up JOA score - preoperative JOA score) $\times$ 100/(29 - preoperative JOA score). All patients completed a 10-point visual analog scale (VAS) as an assessment of their pain. Neurological outcomes, VAS scores, imaging, and postoperative complications of these patients were followed for $\geq 1$ year ( 3 and 6 months and every year after operation; mean 2.4 years, range $1.0-4.5$ years). The human ethics review committee of our institution approved the study protocol, and a signed informed consent was obtained from each patient before enrollment in the study.

\section{Radiological Assessment}

Radiological studies comprised plain lumbopelvic radiographs (neutral, flexion, and extension views), CT scanning (CT/T 8800, General Electric), and high-resolution MRI (1.5 Tesla Signa, General Electric).

On the plain radiographs, we assessed other vertebral collapses (if any), the type of vertebral collapse, intervertebral instability, and sagittal spinopelvic alignment before and after surgery. Each OVC was classified into 1 of 3 different types based on its appearance on the lateral projection in neutral position, as described in previous reports, $4,15,17,22$ including 1 report by our group ${ }^{3,26,27}$ (Fig. 1). In Type 1, or wedge-type collapse, the ratio of the anterior height of the vertebral body to its posterior height was < $60 \%$. Type 2 encompassed flat fracture or vertebra planalike fracture with uniform compression. Type 3 included 


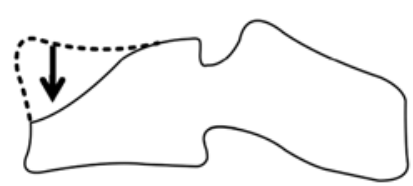

Type 1

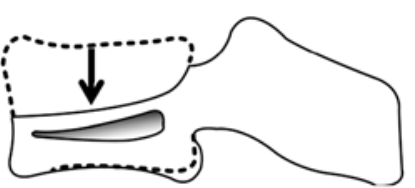

Type 2

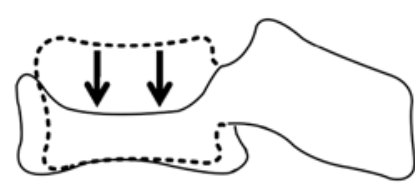

Type 3

FIG. 1. Illustrations showing the 3 types of OVC classified according to appearance on lateral radiographs and MR images. For wedge collapse (Type 1), the ratio of anterior height to posterior height of the vertebral body is less than $60 \%$. Flat or vertebra plana (Type 2) has uniform compression that is often associated with the appearance of intervertebral cleft formation. Concave collapse (Type 3) shows anterior spur, sclerotic changes, or $\mathrm{H}$ shape. Arrows indicate regions of collapse.

concave or $\mathrm{H}$-shaped fracture with anterior spur formation or sclerotic change. Type 2 collapse is often associated with the appearance of intervertebral cleft formation on radiographs or MR images. ${ }^{6,13}$

We assessed the following parameters to determine the spinopelvic alignment ${ }^{11,21}$ : pelvic incidence (PI), defined as the angle between a line joining the center of the upper endplate of S-1 to the axis of the femoral heads and a line perpendicular to the upper endplate of S-1; lumbar lordosis (LL), defined as the angle between tangent lines to the inferior endplates of L-1 and superior endplates of S-1; pelvic tilt (PT), defined as the angle between a vertical line and the line joining the middle of the sacral plate and the axis of the femoral heads; sacral slope (SS), defined as the angle between the endplate of S-1 and a horizontal line. On the sagittal, axial, and coronal CT scans and MRI images, we assessed the presence of foraminal stenosis and canal stenosis (Fig. 2). Bone mineral density (BMD) of the lumbar spine (L1-4) in the posteroanterior projection was measured with dual-energy x-ray absorptiometry (QDR 1000 , Hologic), and the results are expressed in $\mathrm{g} / \mathrm{cm}^{2}$. Plain radiographs and CT scans were followed up 3 and 6 months and every year postoperatively. Two independent observers were responsible for radiological assessment, and both were blinded to the data related to surgery.

\section{Statistical Analysis}

Clinical and radiological findings were assessed by observers independent of the principal surgeons (H.N. and
K.H.). All values are expressed as mean \pm SD. Differences between groups were examined for statistical significance using Wilcoxon signed-rank test or Mann-Whitney U-test. A probability value less than 0.05 was considered to represent a statistically significant difference. All statistical analyses were conducted using the SPSS software (version 15.0, SPSS).

\section{Results \\ Clinical Symptoms and Surgical Outcomes}

Table 1 summarizes the symptoms of the 30 patients with low lumbar OVC stratified by the presence of cleft formation. The majority of patients with cleft formation at the affected vertebra complained of both severe lowback pain $(85.7 \%)$ and radicular pain $(85.7 \%)$, while the incidence of low-back pain was much lower $(25.0 \%)$ in those without cleft formation than those with radicular pain $(93.8 \%)$. The mean JOA score improved significantly from $13.8 \pm 5.5$ preoperatively to $22.4 \pm 5.9$ at follow-up; the mean improvement rate was $61.4 \% \pm 24.8 \%$. The mean VAS pain score improved significantly from $8.1 \pm 1.3$ preoperatively to $3.5 \pm 2.1$ at follow-up. There was no significant difference in JOA score or VAS pain score among those with cleft formation or surgical procedures.

\section{Image Findings in Patients With Low Lumbar OVC}

Assessment of the plain radiographs showed that most patients (25 patients [83.3\%]) presented with old compression fractures at the thoracolumbar level. With regard to
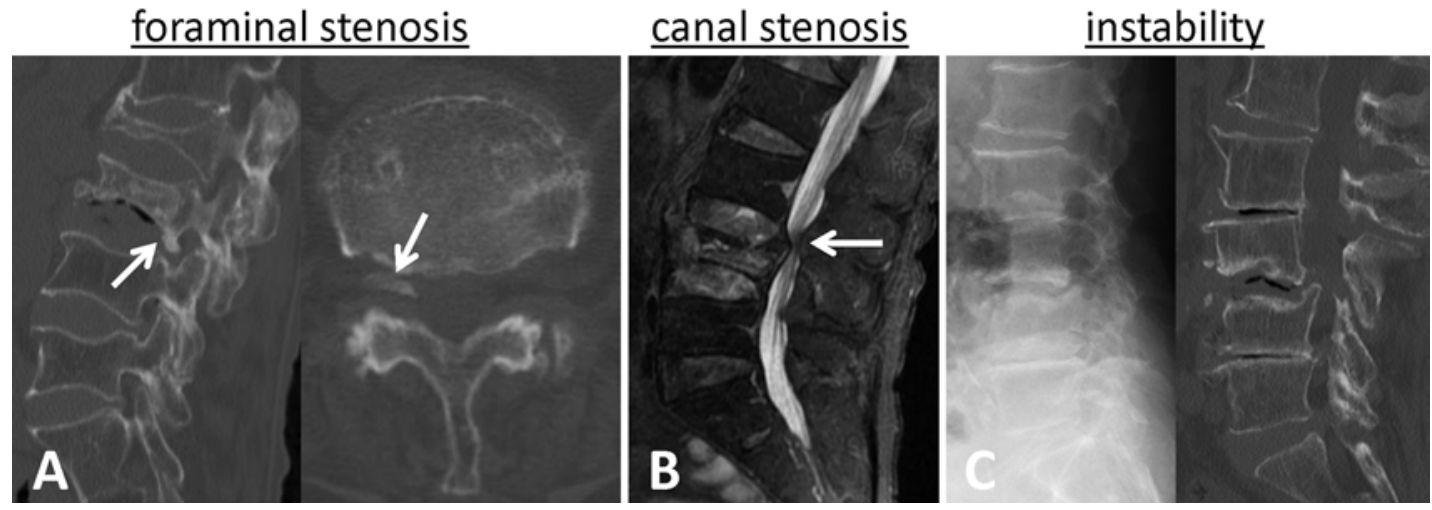

FIG. 2. Representative preoperative radiological images showing foraminal stenosis, canal stenosis, and intervertebral instability. A: Parasagittal (left) and axial (right) CT scans showing foraminal stenosis (arrows). B: T2-weighted MR image showing canal stenosis (arrow). C: Dynamic sagittal radiograph (left) and sagittal CT scan (right) showing intervertebral instability. 
TABLE 1. Symptoms of patients with low lumbar vertebral collapse*

\begin{tabular}{ccr}
\hline \multirow{2}{*}{ Symptom } & \multicolumn{2}{c}{ Cleft Formation in Affected Vertebra } \\
\cline { 2 - 3 } & Present & \multicolumn{1}{c}{ Absent } \\
\hline Low-back pain & $12 / 14(85.7)$ & $4 / 16(25.0)$ \\
\hline Radicular pain & $12 / 14(85.7)$ & $15 / 16(93.8)$ \\
\hline Cauda equina sign & $5 / 14(35.7)$ & $4 / 16(25.0)$ \\
\hline
\end{tabular}

* Data are number of patients/total number of patients (\%).

the type of OVC, none of the patients had Type 1 vertebral collapse (wedge-type), 16 patients had Type 2 (flat-type), and 14 patients had Type 3 (concave or H-shaped type). Fourteen patients (87.5\%) with Type 2 vertebral collapse had cleft formation at the affected vertebra. In 11 patients (36.7\%), intervertebral instability was evident between the upper and affected vertebra. In all of these patients, there was no cleft formation at the affected vertebra; 11 (68.8\%) of 16 patients without cleft formation demonstrated intervertebral instability. Table 2 provides the means and SDs of sagittal spinopelvic alignment before and at the last available follow-up after surgery. LL decreased $\left(16.5^{\circ} \pm\right.$ $\left.10.2^{\circ}\right)$, and a mismatch between PI and LL $\left(30.4^{\circ} \pm 10.0^{\circ}\right)$ was detected in patients with low lumbar OVC. However, it was difficult for the surgical subjects selected in this study to achieve proper spinopelvic alignment after treatment. Examination of CT scans and MR images showed foraminal stenosis in $18(60.0 \%)$ of 30 patients as well as canal stenosis in $24(80.0 \%)$ of 30 of the patients.

\section{Postoperative Complications and Related Factors}

Postoperative complications occurred in 8 patients (26.7\%), including progression of vertebral collapse in 4 and loosening and migration of pedicle screws in 8; these complications developed within 6 months after surgery (Table 3). The migration of pedicle screws occurred in 5 patients after VP + PSF + PLF and in 3 patients after PSF + PLF; most of these postoperative complications occurred before bone union of PLF. Among these patients, 2 showed vertebral collapse progression at adjacent vertebra and kyphotic changes associated with loosened pedicle screws; revision surgeries were performed in both patients. Table 4 provides summary data on sagittal alignment and bone density in relation to postoperative complications.

TABLE 2. Sagittal spinopelvic alignment before and after surgery*

\begin{tabular}{cccc}
\hline Alignment & Before Surgery & Follow-Up & p Value $†$ \\
\hline PI & \multicolumn{2}{c}{$46.3 \pm 6.0$} & - \\
\hline LL & $16.5 \pm 10.2$ & $20.5 \pm 10.2$ & 0.316 \\
\hline PT & $17.4 \pm 7.5$ & $16.4 \pm 7.3$ & 0.387 \\
\hline SS & $28.9 \pm 6.0$ & $29.9 \pm 7.5$ & 0.352 \\
\hline PI - LL & $30.4 \pm 10.0$ & $26.5 \pm 12.7$ & 0.406 \\
\hline
\end{tabular}

* Data are mean angle in degrees \pm SD.

$\dagger$ Based on Wilcoxon signed-rank test.

$\ddagger$ Value is a patient-specific characteristic and remains unchanged after surgery.
TABLE 3. Postoperative complications

\begin{tabular}{lcl}
\hline \multicolumn{1}{c}{ Complication \& Level } & $\begin{array}{c}\text { No. of } \\
\text { Cases }\end{array}$ & $\begin{array}{c}\text { Postop } \\
\text { Period (mos) }\end{array}$ \\
\hline Progression of vertebral collapse & & \\
\hline Affected vertebra & 1 & 6 \\
\hline Adjacent vertebra & 2 & 5 and 6 \\
\hline$\quad$ Remote vertebra & 1 & 3 \\
\hline Loosening and migration of pedicle screw & & \\
\hline Upper level & 8 & $3-6$ \\
\hline
\end{tabular}

There was no change in sagittal spinopelvic alignment after surgery in relation to postoperative complications. However, lumbar spine BMD was significantly lower in patients with postoperative complications $(\mathrm{p}=0.048)$.

\section{Discussion}

The aim of this study was to clarify the clinical and imaging features as well as outcomes of low lumbar OVC and to discuss the appropriate surgical treatment. The major findings of our research were 1) the morphological features of OVC in patients with low lumbar vertebra are flattype (Type 2) and concave or H-shaped type (Type 3) with old compression fracture at the thoracolumbar lesion; 2) intervertebral cleft was evident mainly in Type 2 collapse while intervertebral instability was evident mainly in Type 3 collapse; 3 ) the main clinical symptom in these patients is radicular leg pain originating from foraminal stenosis by retropulsed bony fragments; 4) for patients with cleft formation at the affected vertebra, we recommend VPaugmented short-segment fixation; 5) for patients without cleft formation at the affected vertebra, we recommend PLIF or PLF according to the presence of intervertebral instability; and 6) the high postoperative complication rates could be due to vertebral fragility and/or old age.

OVC is treated surgically in patients with severe and intractable back pain, neurological complications, and/or impending paralysis. Significant clinical challenges must be acknowledged when performing surgical treatment, including the advanced age of the patients, fragility of the vertebra, and presence of serious comorbidities. In this study, the mean age of the patients at surgery was 79.3

TABLE 4. Comparison of patients with and without complications*

\begin{tabular}{llccc}
\hline \multirow{2}{*}{ Alignment } & Surgery & $\begin{array}{c}\text { w/ } \\
\text { Complications }\end{array}$ & $\begin{array}{c}\text { w/o } \\
\text { Complications }\end{array}$ & $\begin{array}{c}p \\
\text { Value }\end{array}$ \\
\hline $\mathrm{PI}$ & - & $45.4 \pm 6.3$ & $46.9 \pm 6.2$ & 0.690 \\
\hline $\mathrm{LL}$ & Before & $21.5 \pm 9.1$ & $16.4 \pm 8.9$ & 0.391 \\
\cline { 2 - 5 } & After & $24.7 \pm 5.9$ & $18.6 \pm 11.6$ & 0.270 \\
\hline $\mathrm{PI}-\mathrm{LL}$ & Before & $26.5 \pm 10.8$ & $31.4 \pm 10.0$ & 0.480 \\
\cline { 2 - 5 } & After & $22.7 \pm 8.1$ & $30.1 \pm 13.0$ & 0.481 \\
\hline $\begin{array}{c}\text { Lumbar spine } \\
\text { BMD }\left(\mathrm{g} / \mathrm{cm}^{2}\right)\end{array}$ & Before & $0.60 \pm 0.08$ & $0.68 \pm 0.05$ & 0.048 \\
\hline
\end{tabular}

* Data are mean angle in degrees \pm SD except where otherwise indicated.

$\dagger$ Based on Mann-Whitney U-test. 
A
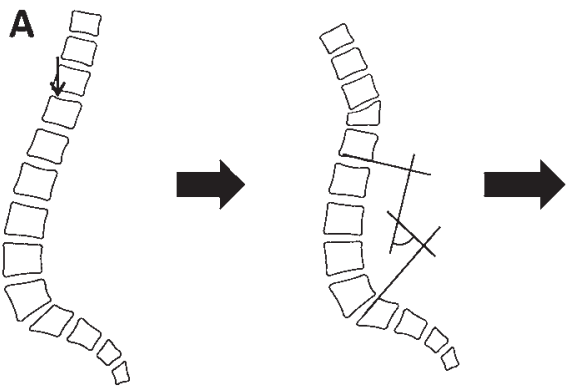

Thoracolumbar vertebral collapse
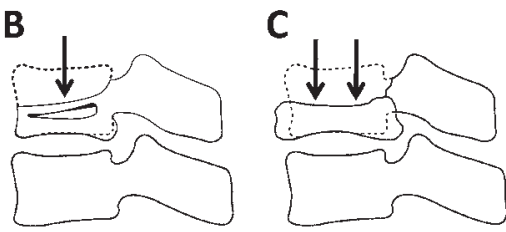

D

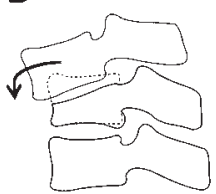

FIG. 3. Illustrations showing pathomechanism of low lumbar OVC. A: Diagrams showing existing thoracolumbar compression and decreasing LL (L1-S1) seen in most patients (left and center), with middle and/or posterior loading to low lumbar spine increasing (right) following spinal alignment changes. B and C: Diagram depicting cases of flat-type (B) and concave or $\mathrm{H}$-shaped type (C) collapse, where foraminal stenosis and/or canal stenosis by the bony fragments are often seen. D: Illustration showing intervertebral instability between affected and upper vertebra often seen in cases of concave or H-shaped type OVC.

years, which is considerably older than patients with thoracolumbar vertebral collapse (70.3 years) described in our previous study. ${ }^{27}$ The average lumbar spine BMD of these patients was $0.64 \mathrm{~g} / \mathrm{cm}^{2}$, indicating poor bone quality. Due to anatomical differences between thoracolumbar and low lumbar lesions with regard to nerve structures and spinal alignment, the treatment strategies for the 2 lesions are also different.

OVC are divided into several types according to their appearance on radiological studies: a wedge-type compression fracture (Type 1) exhibiting progressive kyphosis; a flat-type fracture (Type 2) with osteonecrosis or pseudarthrosis, often exhibiting intervertebral cleft formation; and a concave or $\mathrm{H}$-shaped fracture (Type 3 ) associated with anterior spur or sclerotic changes. In the present study, vertebral collapse was classified into 3 types based on its appearance on preoperative lateral radiographs. We found that the main types of low lumbar OVC were flat-type (Type 2) and concave or H-shaped type (Type 3), not the wedge type (Type 1), as often found in thoracolumbar OVC. The different proportions are probably related to differences in the pathomechanisms of low lumbar OVC and thoracolumbar lesions. Based on the results of the present study, most patients with low lumbar OVC presented with old compression fractures at the thoracolumbar lesion, with decreased LL. This is because the types of OVC between thoracolumbar and low lumbar lesions were different; middle and/or posterior low lumbar spine loading is increased following spinal alignment changes (Fig. 3A). In the flat-type (Type 2) and concave or H-shaped type (Type 3) low lumbar OVC, neurological symptoms result from retropulsed bony fragments causing foraminal stenosis and/or canal stenosis ${ }^{8,19}$ (Fig. 3B and C). In general, foraminal stenosis is classified into anteroposterior stenosis "transverse stenosis" and craniocaudal stenosis "vertical stenosis." Protrusion of the posterior wall of the vertebral body and facet hypertrophy can cause anteroposterior compression with or without canal stenosis. On the other hand, reduced vertebral body height with bony defect at the caudal side of the affected vertebra is detected in some cases, and when under the upper pedicle can cause craniocaudal compression in a fractured spine. ${ }^{20}$ Some cases of concave or $\mathrm{H}$ shaped type (Type 3) vertebra showed sclerotic changes, with higher chance of intervertebral instability between affected and upper vertebra (Fig. 3D). In these situations,

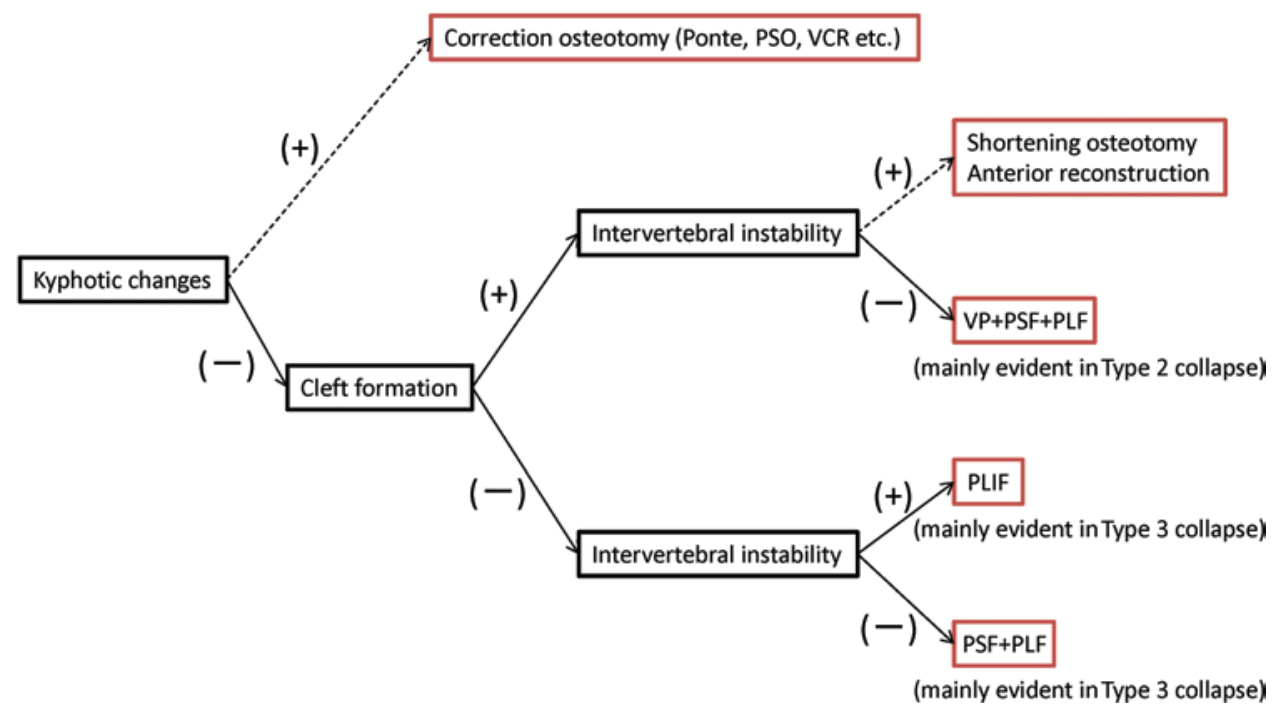

FIG. 4. Schematic showing the surgical strategy for low lumbar OVC. The procedure used was based on the following factors: kyphotic changes in the lumbar spine, intervertebral cleft formation, and intervertebral instability. The number of patients in procedures denoted by the dotted arrows was small. PSO = pedicle subtraction osteotomy. Figure is available in color online only. 

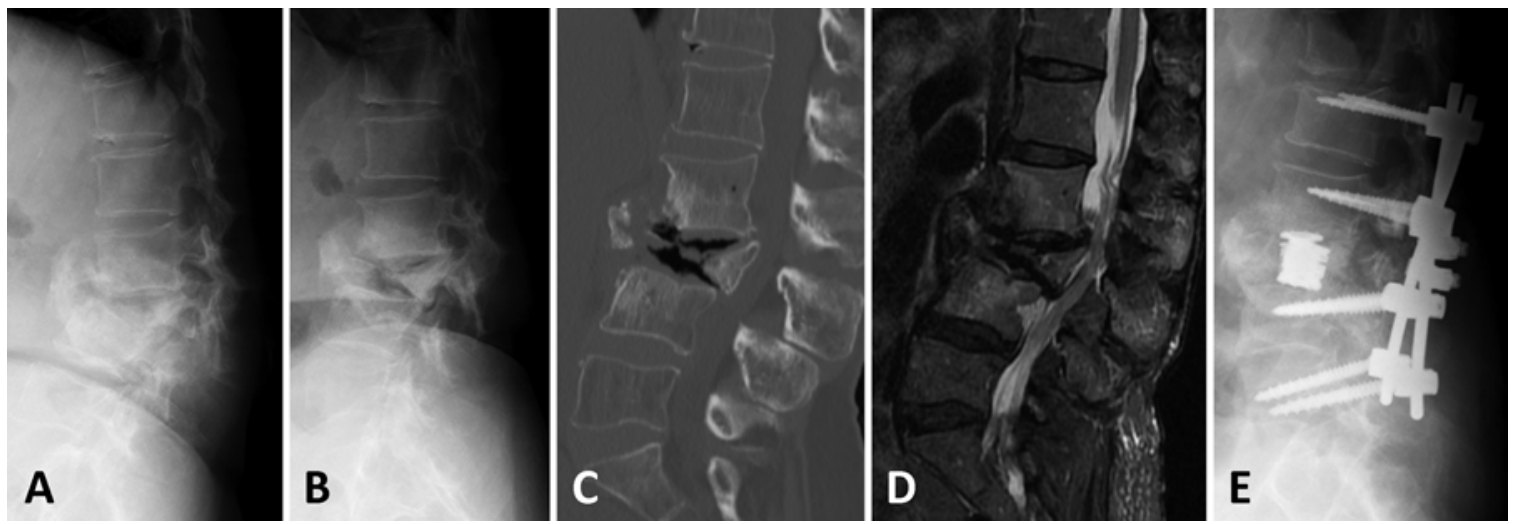

FIG. 5. Case of a 70-year-old woman with burst fracture at the anterior column of L-3 and local kyphosis due to OVC is representative of correction osteotomy for local kyphotic changes in the lumbar spine. A and B: Dynamic radiographs showing vertebral instability. C: Radiograph showing the vacuum phenomenon in both L2-3 and L3-4 discs. D: Sagittal T2-weighted MR image showing compression of the dural tube between L2-3 and L3-4 levels. E: Radiograph taken after VCR with vertebral body replacement and PSF (L1-5) was performed.

direct decompression of nerve tissue and reconstruction of the spinal column is required.

With regard to the surgical strategy for thoracolumbar OVC, from a biomechanical point of view, when only the anterior column is injured among the three Denis-defined columns and characteristic progressive kyphosis is evident, combined anterior decompression and fusion is the ideal choice., ${ }^{9,27}$ However, for low lumbar OVC, the posterior approach may be the ideal surgical option because the main pathology, fractured bone, is located at the middle and posterior parts of the affected vertebra; the decompression procedure for lumbar canal and intervertebral foramen is easier and the anterior approach to low lumbar spine is difficult; and an anterior approach poses risks to abdominal organs and vessels, which could cause serious complications in elderly patients. Figure 4 shows the surgical strategies for low lumbar OVC based on our observations and experience in the present study. Although our primary method of short fusion using a posterior approach was considered the less stressful or less invasive operation to resolve back pain and radicular leg pain, correction osteotomy and long fusion were performed in a few cases to restore spine alignment ${ }^{18}$ (Figs. 4 and 5). Although most cases of low lumbar OVC presented decreased LL, they rarely exhibited kyphotic changes since the ratio of wedge-type collapse was low. For patients with cleft formation at the affected vertebra, VP + PS + PLF are recommended (Figs. 4 and 6). In our previous study on thoracolumbar OVC, we reported that VP-augmented short-segment fixation seems to offer immediate spinal stability, and the effect seemed equivalent to that of anterior reconstruction. ${ }^{26,28}$ Similar to low lumbar OVC,
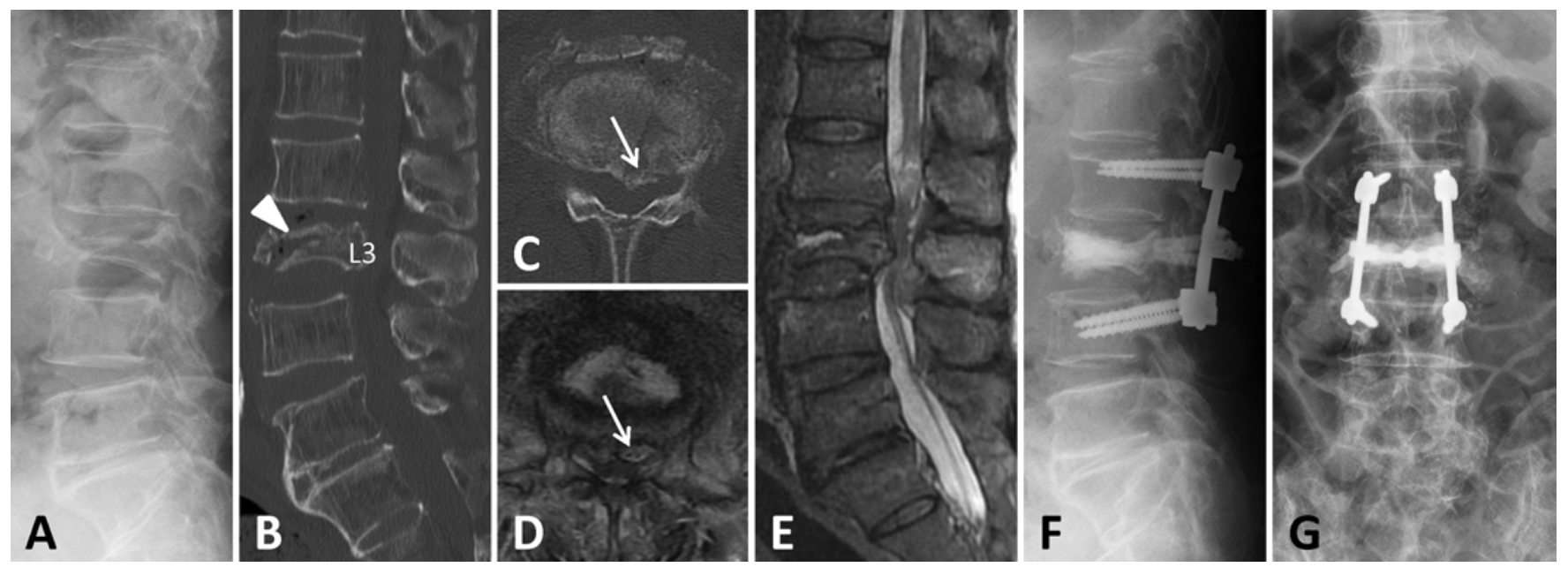

FIG. 6. Case of an 80-year-old man with L-3 flat-type collapse and intervertebral cleft formation, who presented with severe lowback pain and bilateral radicular leg pain, is representative of patients with cleft formation at affected vertebra. A: Preoperative sagittal radiograph showing L-3 collapse (flat-type). B-E: Preoperative parasagittal (B) and axial (C) CT scans and axial (D) and sagittal (E) T2-weighted MR images showing L-3 collapse (arrowhead) with intervertebral cleft formation and canal stenosis by bony fragments (arrows) that had retropulsed into the spinal canal and can be seen between L2-3 and L3-4. F and G: Postoperative sagittal (F) and coronal (G) radiographs showing VP at L-3 with PSF and PLF (L2-4). 

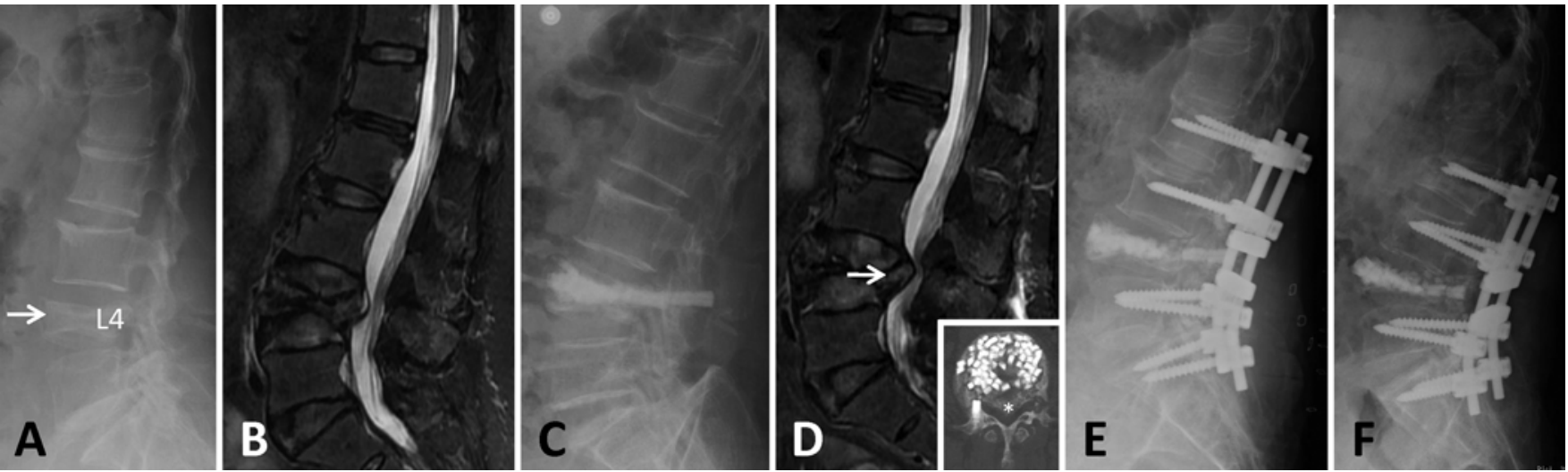

FIG. 7. Case of a 74-year-old woman with L-4 flat-type collapse and intervertebral cleft formation, who is representative of patients who required revision surgery. $A$ and $B$ : Preoperative lateral plain radiograph $(A)$ and sagittal T2-weighted MR image $(B)$ showing flat-type collapse (arrow) and an intervertebral cleft, respectively. C: Postoperative radiograph showing the result of initial surgery, VP at L-4. D: Sagittal T2-weighted MR image and axial CT scan (inset) showing evidence of progression of collapse of the affected vertebra (arrow) 6 months after the initial surgery and compression of the dural tube between L3-4 and L4-5 levels produced by bony fragments retropulsed into the spinal canal (asterisk). E: Radiograph showing the results of revision surgery, decompression with PSF (L2-S1) and PLF (L3-5). F: Radiograph showing progression of collapse of the upper end of the fixation and adjacent vertebra with loosened pedicle screw at 5 months after revision surgery.

VP is available for the stability of the collapsed vertebra. However, VP without short fusion (only VP) is not recommended because the load on middle and/or posterior lesions of the affected vertebra increased in this group, and collapse of the affected vertebra could easily progress. The clinical outcome of VP without short fusion for patients with vertebral body height loss of $\geq 70 \%$ is poor in most cases with Type 2 or Type 3 collapse. ${ }^{1}$ Figure 7 presents 1 of the 2 cases who required revision surgery. In this case, 6 months after VP without fusion for OVC at L-4, the L-4 vertebra recollapsed where retropulsed bony fragments in the spinal canal generated neurological deficits. Revision surgery including decompression + PSF (L2-S1) + PLF was performed, but the upper end of the fixation and adjacent vertebra collapsed following screw loosening. In patients without cleft formation, the affected vertebra often showed sclerotic changes. In cases without evidence of intervertebral instability on radiological images, PSF (screw also inserted in the affected vertebra) + PLF are recommended (Figs. 4 and 8). It is advantageous in such cases to insert pedicle screws in the affected vertebra because most of these vertebrae present sclerotic changes, providing good anchor for the spine. In cases with intervertebral instability, the upper vertebra is usually slipped; therefore, PLIF is recommended (Figs. 4 and 9). The PLIF procedure provides excellent clinical outcome for both canal stenosis and foraminal stenosis. ${ }^{30}$

The mean neurological score improved significantly postoperatively; however, the rate of postoperative complications (26.7\%), mainly related to the instrumenta-
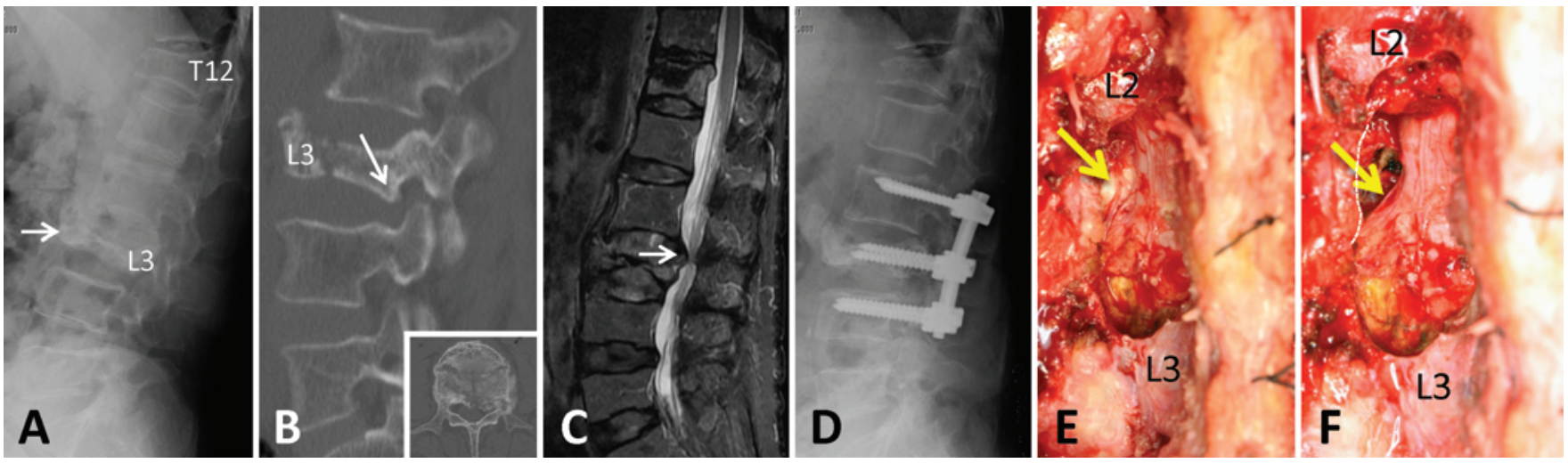

FIG. 8. Case of a 73-year-old woman with L-3 concave type collapse, who is representative of patients with concave sclerotic collapse without intervertebral instability. A: Preoperative lateral plain radiograph showing L-3 collapse (arrow). B: Preoperative CT scan showing foraminal stenosis by bony fragments retropulsed into the intervertebral foramen (arrow). Axial view (inset). C: Preoperative sagittal T2-weighted MR image showing canal stenosis at the L2-3 level (arrow). D: Radiograph showing decompression with PSF (L3-5) with sclerotic changes affecting the vertebra and PLF (L3-5). E: Intraoperative photograph showing bony fragments that had retropulsed into the intervertebral foramen and compressed left L-3 root (yellow arrow). F: Photograph showing decompression of the left L-3 root (yellow arrow) performed via medial foraminotomy. Figure is available in color online only. 

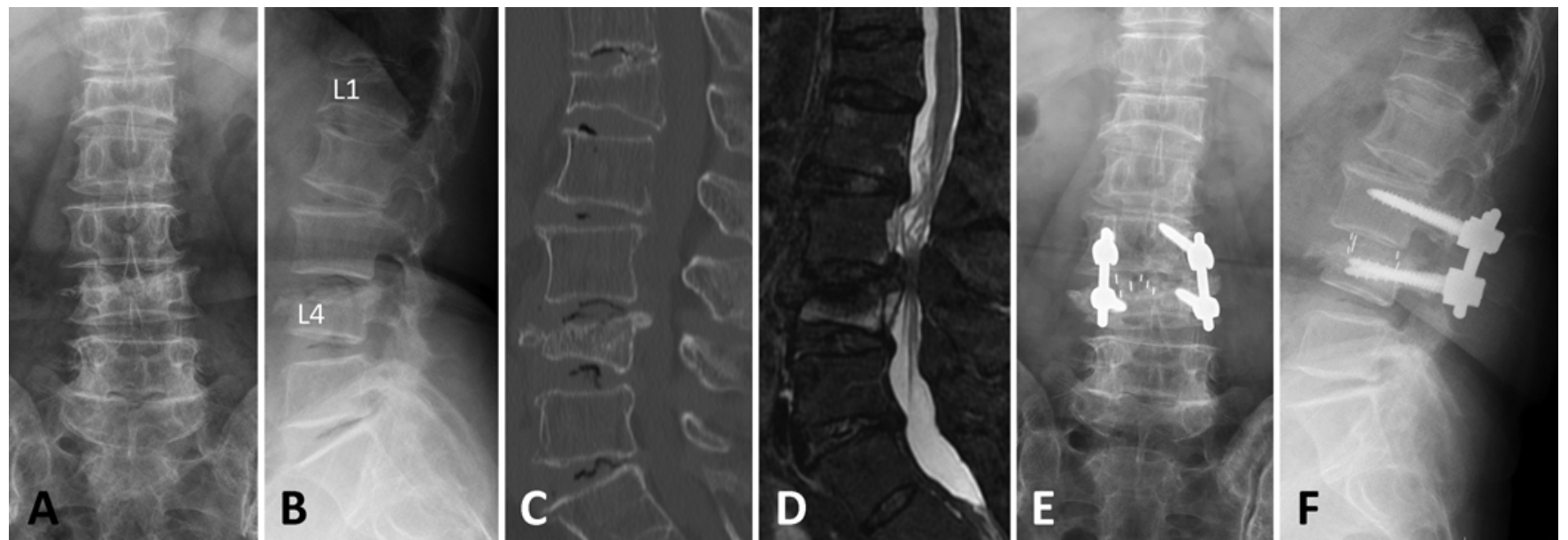

FIG. 9. Case of a 73-year-old woman with L-4 flat-type collapse and intervertebral instability between L-3 and L-4, who is representative patients with intervertebral instability. A and B: Preoperative anteroposterior $(A)$ and lateral plain $(B)$ radiographs showing that the intervertebral instability included lateral shift. C: Preoperative CT scan showing upper endplate destruction and sclerotic changes in the L-4 vertebra. D: Preoperative sagittal MR image showing canal stenosis at the L3-4 level. E and F: Radiographs showing the results of PLIF at L3-4.

tion, was not low in this study. Our results indicate that the surgical procedure of short fusion with posterior approach cannot correct the mismatch between LL and PI. However, the sagittal spinopelvic alignment was not different between patients with and without postoperative complications in the present study, while the lumbar spine BMD was significantly lower in patients with postoperative complications. Although the PSF method can achieve strong spinal fixation, the severity of osteoporosis plays a major role in the pullout force of the screws. ${ }^{23,24}$ These results indicate that it is not necessary to correct the sagittal alignment in patients with low lumbar OVC, except in cases requiring long fusion or multiple site of fixation for accepting lesser degrees of deformity correction and avoiding ending instrumentation within kyphotic segments or when the severity of osteoporosis is very high. ${ }^{7,14}$ To avoid these postoperative complications, it may be necessary to implement some augmentation, such as use of a supplemental hook system or sublaminar cables combined with standard short segment PSF, ${ }^{24}$ together with treatment of osteoporosis, for example, using parathyroid hormone (PTH). ${ }^{16}$

This study had certain limitations. First, patients in this series were elderly, and it was impossible to follow 11 of 30 patients because 9 patients died of other diseases and 2 patients could not visit our hospital postoperatively due to excessive travel distance and/or physical disability. We could only check the final follow-up data for these patients from the medical records or telephone interview. Imaging and mean follow-up period in these patients was only 2.4 years (1.0-4.5 years). Another limitation was the use of PTH and/or some augmentations in surgeries in the patients seen in recent years. It is possible this study underrepresents the true failure or complication rate. Second, since the study was a small retrospective case series, multivariate analysis of each parameter was not possible. For these limitations, further investigation of a large number of cases is required to assess the surgical outcome and to design a validated algorithm for low lumbar OVC. However, since a few reported studies have examined patients with low lumbar OVC, the present study on the clinical status, neurological symptoms, radiological findings of such patients, no doubt, provides important information regarding surgical strategies and outcome.

\section{Conclusions}

The morphological features of OVC in patients with low lumbar vertebra are flat-type and concave or $\mathrm{H}$-shaped type. In these, foraminal stenosis, canal stenosis, and/or intervertebral instability are found, and severe radicular leg pain may occur. For these patients, decompression and short fusion surgery via a posterior approach can improve the neurological symptoms. However, these patients present at advanced age, with poor bone quality and other comorbidities, where the rate of postoperative complications related to the instrumentation is less than ideal. Surgical strategy and treatment of osteoporosis are both important in these patients.

\section{References}

1. Alvarez L, Alcaraz M, Pérez-Higueras A, Granizo JJ, de Miguel I, Rossi RE, et al: Percutaneous vertebroplasty: functional improvement in patients with osteoporotic compression fractures. Spine (Phila Pa 1976) 31:1113-1118, 2006

2. Arciero RA, Leung KY, Pierce JH: Spontaneous unstable burst fracture of the thoracolumbar spine in osteoporosis. A report of two cases. Spine (Phila Pa 1976) 14:114-117, 1989

3. Baba H, Maezawa Y, Kamitani K, Furusawa N, Imura S, Tomita K: Osteoporotic vertebral collapse with late neurological complications. Paraplegia 33:281-289, 1995

4. Denis F: The three column spine and its significance in the classification of acute thoracolumbar spinal injuries. Spine (Phila Pa 1976) 8:817-831, 1983

5. Frost HM: Clinical management of the symptomatic osteoporotic patient. Orthop Clin North Am 12:671-681, 1981

6. Hasegawa K, Homma T, Uchiyama S, Takahashi H: Vertebral 
pseudarthrosis in the osteoporotic spine. Spine (Phila Pa 1976) 23:2201-2206, 1998

7. Hu SS: Internal fixation in the osteoporotic spine. Spine (Phila Pa 1976) 22 (24 Suppl):43S-48S, 1997

8. Jenis LG, An HS: Spine update. Lumbar foraminal stenosis. Spine (Phila Pa 1976) 25:389-394, 2000

9. Kaneda K, Asano S, Hashimoto T, Satoh S, Fujiya M: The treatment of osteoporotic-posttraumatic vertebral collapse using the Kaneda device and a bioactive ceramic vertebral prosthesis. Spine (Phila Pa 1976) 17 (8 Suppl):S295-S303, 1992

10. Kim KT, Suk KS, Kim JM, Lee SH: Delayed vertebral collapse with neurological deficits secondary to osteoporosis. Int Orthop 27:65-69, 2003

11. Labelle H, Roussouly P, Berthonnaud E, Transfeldt E, O'Brien M, Chopin D, et al: Spondylolisthesis, pelvic incidence, and spinopelvic balance: a correlation study. Spine (Phila Pa 1976) 29:2049-2054, 2004

12. Lee YL, Yip KM: The osteoporotic spine. Clin Orthop Relat Res (323): 91-97, 1996

13. Maldague BE, Noel HM, Malghem JJ: The intravertebral vacuum cleft: a sign of ischemic vertebral collapse. Radiology 129:23-29, 1978

14. McLain RF, Sparling E, Benson DR: Early failure of shortsegment pedicle instrumentation for thoracolumbar fractures. A preliminary report. J Bone Joint Surg Am 75:162-167, 1993

15. Mochida J, Toh E, Chiba M, Nishimura K: Treatment of osteoporotic late collapse of a vertebral body of thoracic and lumbar spine. J Spinal Disord 14:393-398, 2001

16. Ohtori S, Inoue G, Orita S, Yamauchi K, Eguchi Y, Ochiai N, et al: Comparison of teriparatide and bisphosphonate treatment to reduce pedicle screw loosening after lumbar spinal fusion surgery in postmenopausal women with osteoporosis from a bone quality perspective. Spine (Phila Pa 1976) 38:E487-E492, 2013

17. Peh WC, Gilula LA, Peck DD: Percutaneous vertebroplasty for severe osteoporotic vertebral body compression fractures. Radiology 223:121-126, 2002

18. Saita K, Hoshino Y, Higashi T, Yamamuro K: Posterior spinal shortening for paraparesis following vertebral collapse due to osteoporosis. Spinal Cord 46:16-20, 2008

19. Sasaki M, Aoki M, Nishioka K, Yoshimine T: Radiculopathy caused by osteoporotic vertebral fractures in the lumbar spine. Neurol Med Chir (Tokyo) 51:484-489, 2011

20. Sasaki Y, Aoki Y, Nakajima A, Shibata Y, Sonobe M, Takahashi K, Ohtori S, Nakagawa K: Delayed neurologic deficit due to foraminal stenosis following osteoporotic late collapse of a lumbar spine vertebral body. Case Rep Orthop 2013:682075, 2013

21. Schwab F, Lafage V, Patel A, Farcy JP: Sagittal plane considerations and the pelvis in the adult patient. Spine (Phila Pa 1976) 34:1828-1833, 2009

22. Shikata J, Yamamuro T, Iida H, Shimizu K, Yoshikawa J: Surgical treatment for paraplegia resulting from vertebral fractures in senile osteoporosis. Spine (Phila Pa 1976) 15:485-489, 1990
23. Soshi S, Shiba R, Kondo H, Murota K: An experimental study on transpedicular screw fixation in relation to osteoporosis of the lumbar spine. Spine (Phila Pa 1976) 16:13351341,1991

24. Sudo H, Ito M, Abumi K, Kotani Y, Takahata M, Hojo Y, et al: One-stage posterior instrumentation surgery for the treatment of osteoporotic vertebral collapse with neurological deficits. Eur Spine J 19:907-915, 2010

25. Taneichi H, Kaneda K, Oguma T, Kokaji M: [Risk factor analysis for osteoporotic vertebral collapse and pseudarthrosis.] Rinsyo Seikeigeka 37:437-442, 2002 (Jpn)

26. Uchida K, Kobayashi S, Matsuzaki M, Nakajima H, Shimada $\mathrm{S}$, Yayama T, et al: Anterior versus posterior surgery for osteoporotic vertebral collapse with neurological deficit in the thoracolumbar spine. Eur Spine J 15:1759-1767, 2006

27. Uchida K, Kobayashi S, Nakajima H, Kokubo Y, Yayama T, Sato R, et al: Anterior expandable strut cage replacement for osteoporotic thoracolumbar vertebral collapse. J Neurosurg Spine 4:454-462, 2006

28. Uchida K, Nakajima H, Yayama T, Miyazaki T, Hirai T, Kobayashi S, et al: Vertebroplasty-augmented short-segment posterior fixation of osteoporotic vertebral collapse with neurological deficit in the thoracolumbar spine: comparisons with posterior surgery without vertebroplasty and anterior surgery. J Neurosurg Spine 13:612-621, 2010

29. Watanabe K, Hikata T, Iwanami A, Hosogane N, Ishii K, Nakamura M, et al: [Radiographic characteristics and surgical results of lumbar spinal stenosis associated with compression fracture.] J East Jpn Orthop Traumatol 25:137-141, 2013 (Jpn)

30. Watanabe K, Yamazaki A, Morita O, Sano A, Katsumi K, Ohashi M: Clinical outcomes of posterior lumbar interbody fusion for lumbar foraminal stenosis: preoperative diagnosis and surgical strategy. J Spinal Disord Tech 24:137-141, 2011

\section{Disclosure}

The authors report no conflict of interest concerning the materials or methods used in this study or the findings specified in this paper.

\section{Author Contributions}

Conception and design: Nakajima, Uchida. Acquisition of data: Nakajima, Honjoh, Sakamoto, Kitade. Analysis and interpretation of data: Nakajima, Honjoh, Sakamoto. Drafting the article: Nakajima. Critically revising the article: Uchida. Reviewed submitted version of manuscript: Uchida, Baba. Statistical analysis: Nakajima, Honjoh. Administrative/technical/material support: Uchida. Study supervision: Baba.

\section{Correspondence}

Hideaki Nakajima, Department of Orthopaedics and Rehabilitation Medicine, University of Fukui Faculty of Medical Sciences, 23-3 Matsuoka Shimoaizuki, Eiheiji, Fukui 910-1193, Japan. email: nhideaki@u-fukui.ac.jp. 